\title{
A Virtual File System for On-Demand Processing of Multidimensional Datasets
}

\author{
Arthur Wetzel \\ Pittsburgh Supercomputing Center \\ Carnegie Mellon University \\ Pittsburgh, PA 15213 \\ 01-412-268-3912 \\ awetzel@psc.edu
}

\author{
Jennifer Bakal \\ Pittsburgh Supercomputing Center \\ Carnegie Mellon University \\ Pittsburgh, PA 15213 \\ 01-412-268-6340 \\ jennb@psc.edu
}

\author{
Markus Dittrich \\ BioTeam Inc. \\ Middleton, MA 01949 \\ 01-412-345-8040 \\ markus@bioteam.net
}

\begin{abstract}
Diverse areas of science and engineering are increasingly driven by high-throughput automated data capture and analysis. Modern acquisition technologies, used in many scientific applications (e.g., astronomy, physics, materials science, geology, biology, and engineering) and often running at gigabyte per second data rates, quickly generate terabyte to petabyte datasets that must be stored, shared, processed and analyzed at similar rates. The largest datasets are often multidimensional, such as volumetric and time series data derived from various types of image capture. Costeffective and timely processing of these data require system and software architectures that incorporate on-the-fly processing to minimize I/O traffic and avoid latency limitations. In this paper we present the Virtual Volume File System, a new approach to on-demand processing with file system semantics, combining these principles into a versatile and powerful data pipeline for dealing with some of the largest $3 \mathrm{D}$ volumetric datasets. We give an example of how we have started to use this approach in our work with massive electron microscopy image stacks. We end with a short discussion of current and future challenges.
\end{abstract}

\section{CCS Concepts}

- Software and its engineering $\rightarrow$ Middleware. • Information systems $\rightarrow$ Deduplication. • Networks $\rightarrow$ In-network processing. - Applied computing $\rightarrow$ Life and medical sciences.

\section{Keywords}

active storage file system; multidimensional data processing; near-data computing; data sharing; data duplication; hierarchical data storage.

\section{VIRTUAL VOLUME FILE SYSTEM}

In conventional data-processing pipelines, raw data are captured, stored and subsequently processed any number of times in order to, e.g., apply different types of transformations or feed

\footnotetext{
Permission to make digital or hard copies of part or all of this work for personal or classroom use is granted without fee provided that copies are not made or distributed for profit or commercial advantage and that copies bear this notice and the full citation on the first page. Copyrights for third-party components of this work must be honored. For all other uses, contact the Owner/Author.

Copyright is held by the owner/author(s).

XSEDE16, July 17-21, 2016, Miami, FL, USA.

ACM 978-1-4503-4755-6/16/07

DOI=http://dx.doi.org/10.1145/2949550.2949656
}

into different consumer applications (Figure 1A). The separation of processing from the final analysis simplifies application program development and enables reuse of standard processing capabilities (such as intensity, contrast and geometry corrections for image data) that can then be shared by many different application codes.
A

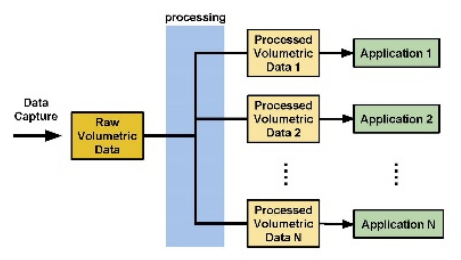

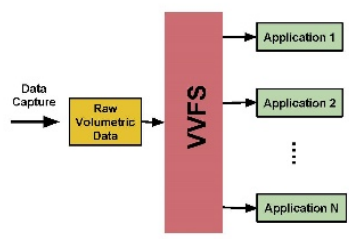

Figure 1. Schematic depiction of (A) conventional and (B) VVFS data flow in the presence of $\mathbf{N}$ different data processing stages. The conventional approaches often replicate intermediate data to $\mathbf{N}$ times original size, while the VVFS approach in many cases can work with one copy of raw data.

However, this conventional approach has a number of severe limitations particularly when scaling toward larger and larger raw datasets. Any variation of processing parameters results in implicit data duplication since processed renditions carry forward both the information from the original data plus the effects of the chosen processing algorithms. In collaborative scenarios, processed files often have to be transferred back and forth over the network between geographically distinct locations, from acquisition to data processing sites and back and on to other collaborators for further analysis and/or additional processing. Further, conventional processing of entire datasets in this fashion also incurs long $\mathrm{I} / \mathrm{O}$ and processing delays. Both data transfer and data duplication become rate-limiting as data sizes increase. With datasets in the multi-terabyte range, the need to maintain multiple intermediate file sets while accurately tracking previous transformations presents difficult storage and data management challenges. At the petabyte scale this data duplication paradigm will not be sustainable.

Our Virtual Volume File-System (VVFS) approach (Figure 1B) overcomes the most severe shortcomings of current conventional data processing pipelines. VVFS makes processed data available to end-user applications in the form of virtual files which are accessed in the same way as pre-existing real files (e.g. via open, seek and read). Virtual files are not pre-instantiated, do not reside 
on disk, and their content is dynamically generated in a near-data fashion only when end-user applications access them. Conceptually, this is similar to virtual memory page fault handling, the /proc file system in Linux, or file systems for accessing compressed data which (de)compress data only as it is accessed. Even though we present VVFS as a client-server architecture in which final applications may be remotely mounted from distant sites, the server and client may also run on the same machine.

When users access virtual files, VVFS uses the access parameters such as filename, seek position and other specifications, to invoke computations that generate the required virtual file content on the fly. Each virtual file access typically involves random access to stored raw data to gather the required data regions followed by piecewise streaming computations, using either CPU and/or GPGPU near-data processing, to generate and deliver transformed data blocks (Figure 2). Similarly to UNIX pipes, the transformed data are not stored on disk but are passed directly to calling applications through in-memory buffers. In many situations the computations for final image processing can be done more quickly than reading existing files relative to the speed of disk I/O. This is particularly true when applications are accessing localized subsets within a large dataset. In our VVFS concept every I/O operation is an opportunity to trade storage for on-thefly computing while data may already be in memory.

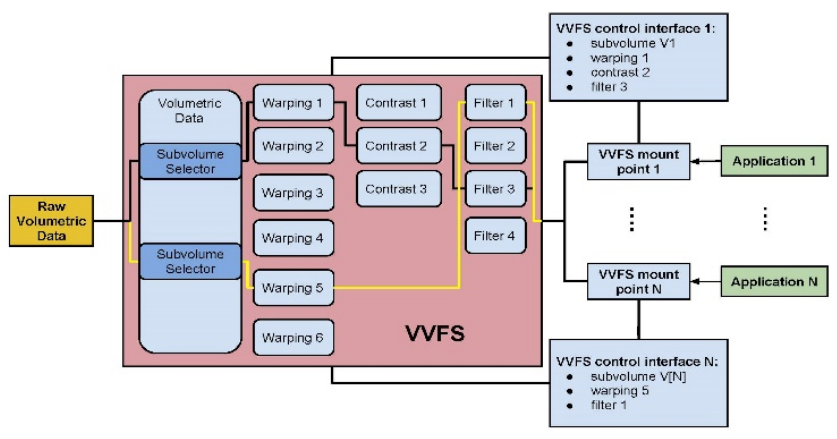

Figure 2. Schematic of pipelined near-data VVFS workflow. Each VVFS mount point delivers dynamic content that is generated on-demand from pipe-like data transform specifications, file names, and file seek position. The transform operations leverage near-data parallel CPU and/or GPGPU computing according to users' available VVFS server configuration.

Various types of active processing with similarities to VVFS have been suggested to improve data-intensive application performance. Argonne National Laboratory has used active storage models to demonstrate the performance benefits obtained using compute power co-located with the data store rather than on separate client systems [1]. These benefits derive largely from reduced network traffic between storage servers and compute nodes. The Active Storage work from the Pacific Northwest National Laboratory improves parallel I/O performance by leveraging idle CPU and GPU cycles within the nodes of large Lustre file servers [2]. The Active Data Repository (ADR) and the Data Cutter by Saltz et al. were developed to leverage parallel computing to enable efficient querying of large multidimensional databases [3,4].

Our VVFS follows similar near-data computing strategies by moving computing into the file system data stream. The key advantages of VVFS for our needs are its ability to leverage characteristics of different data storage layouts to accelerate nonlinear data access, its ability to insert both parallel CPU and GPGPU computing into the workflow, and, by virtue of its virtual file interface, the ability to seamlessly execute within complex application workflows without the need to explicitly store intermediate disk files.

The guiding principles behind our near-data VVFS approach are listed below:

- $\quad$ Bring near-data processing, including large scale parallelism and GPGPU computing, into data analysis and processing workflows to eliminate data duplication and redundant storage and reduce latency.

- $\quad$ Provide a flexible, pipelined data workflow model.

- Minimize data transfer by working directly from local data when possible.

- $\quad$ Minimize delays between data capture and end-user analyses.

- $\quad$ Provide user applications with the conventional (yet virtual) file interfaces they expect.

- Provide an API for specifying on-the-fly computational procedures from raw to virtual files to enable easy deployment of custom written processing routines.

\subsection{Elimination of Data Duplication}

During data analysis, processing steps often require frequent data adjustments. For image based data these may be noise filtering, corrections to intensity and contrast of images, reorientation of data geometry and other aspects that are described by both discrete and continuous transformations. Each of these adjustments typically generates intermediate datasets that may be as large as the original raw data. Storage of this intermediate data on a filesystem creates overhead for both writing by the data producer and reading by the consumer application. In many cases the time required for file $\mathrm{I} / \mathrm{O}$ is large relative to the computations underlying each transformation, particularly when parallel and GPGPU processing are available. Recent rapid increases in computation speeds relative to much slower improvements in disk bandwidth and access times highlight the importance of reducing $\mathrm{I} / \mathrm{O}$ traffic by trading explicit storage for computational regeneration of (intermediate) data.

\subsection{Pipelined Data Flows}

Many scientific analyses are broken into series of steps with differing levels of computational intensity and data access requirements. Conventionally, after initial data acquisition, these steps are linked by data flow through one or typically many layers of intermediate files that store outputs from one processing stage for use as inputs to later stages. In high-performance computing systems, which are typically based on Linux or UNIX operating systems, file semantics are also applied to data flow through pipes and network links. Pipes, one of the key innovations of UNIX, provide a very effective means to organize sequential data flows when intermediate results do not have to be saved or reused and allow users to insert custom routines and standard utilities into the pipeline. This allows users to quickly and flexibly assemble custom processing solutions without the need for explicit intermediate file storage so long as the task can be expressed within a sequential stream paradigm and fits into system memory. Unfortunately, many types of scientific processing require non sequential data access and reuse of intermediate data in ways that 
can not be handled with pipe streams. VVFS overcomes these shortcomings by providing random access and memory mapped operations for user applications while also permitting cascade operation with multiple levels of VVFS processing and intermixing with conventional pipes and or files (Figure 2). In cascade operation, any particular VVFS process can freely access virtual content from earlier stages. For example, a final stage volume rendering or cut plane operation may access a virtual volume defined by another VVFS operation that may in turn build the volume by warping multiple raw images.

\subsection{Minimization of Data Transfer}

Analyses commonly applied to multidimensional data have a wide variety of access and data transfer requirements depending on the portion and type of data needed for a particular task. In order to reduce data traffic to analysis clusters, task aware data structures and adaptive processing within the data servers can be very useful. For example, in projective volume rendering or maximum intensity projection, an entire dataset may be involved in generating a single 2-dimensional view. This is a clear case where near-data processing can avoid transferring entire volumes to client applications and instead directly deliver the much smaller 2dimensional renderings. Importantly, metadata describing the transformations from raw data to processed virtual file content are usually orders of magnitude smaller than the raw data itself. This metadata can be efficiently exchanged over networks and stored to represent and track variants of the processing operations.

\subsection{Minimization of Delays between Data Capture and End Use}

An important use case for VVFS is to enable workflows which provide much quicker initial data analysis by end users than has been conventional practice. For example, with connectomics electron microscopy datasets, image registration is a critical preprocessing step to facilitate analysis of neural pathways. The capture of large volumes takes months, and complete registration also takes many iterations over similar time spans. Traditionally, one had the choice of doing a tedious analysis of raw or roughly aligned data to be remapped later or waiting until full alignment completed. VVFS provides a third option via on-the-fly local registration in which users can immediately access virtual files covering regions that have not yet been registered by the global process. As long as the corresponding raw data exists, the VVFS can preemptively compute high accuracy section-to-section alignments as content for the virtual files, allowing users to follow arbitrary spontaneous analyses without pre-planning or assistance from the alignment specialists.

\subsection{Provide Existing Applications with Conventional File System Access}

A natural solution for structuring VVFS output is via a directory tree with subdirectories and virtual files corresponding to processed data that mirror the content and naming methods used for intermediate files in previous practice. When a virtual file is accessed through the mount point, VVFS invokes the proper processing steps to dynamically generate its content (Figure 2). Unlike sequential pipes, VVFS will support random access in cases when only part of a virtual file's content has to be generated to satisfy a specific read request. In this way users who may be doing spot quality control checks of a large dataset or regional analyses would automatically invoke only the processing and data accesses needed for the immediate task. Future optimizations of the VVFS will provide caching and look ahead processing for sequential or other common access patterns.
A second approach for delivering VVFS output would use pathnames to encode and describe the VVFS processing pipeline corresponding to a particular file access request. This is similar to generating dynamic web content based on parameters embedded in URLs, such that any number of parameters, including floating point variables, can be encoded. The meaning of these parameter fields can be implied by position or by field tag names as usually supplied after the '?' delimiter in dynamic URLs [5].

A third potential access method would apply content-specific interpretations to random access operations. This would, e.g., allow large $3 \mathrm{D}$ volumes to be treated as one large virtual file with the region of interest determined by seek position within the file. The description of VVFS processing could be provided through IOCtl system calls or via writable virtual control files which dynamically alert the VVFS to changes in processing parameters. While these modes would require user applications to be VVFS aware we expect that the control component responsible for sending parameters and commands to VVFS can in most cases be isolated from the main application code

\subsection{Provide User Customizable Raw Data Processing into Virtual Files}

We envision a number of alternatives for user control of VVFS processing. One method is suggested by a tool named ScriptFS [6] which uses executable scripts to generate on demand content and thus illustrates the use of small prestored computational descriptions to direct the generation of potentially much larger virtual file content. VVFS generalizes this method to allow parameterized control files that can be specified on groups of files and/or entire directory trees in a uniform way. These operations include the execution of any user level application utilities. To improve speed we are also developing VVFS internal parallel CPU and GPGPU implementations of common functionality such as data block extraction, arbitrary orientation cut-plane and slab generation, image warping and interpolation. Future work will develop a plugin framework for easy insertion of user coded functions.

\section{VVFS IMPLEMENTATION}

Although VVFS capabilities could be implemented as a kernel space device driver, we decided instead to leverage the FUSE (Filesystem in User Space) technology [7]. FUSE is cross platform and allows fully functional file systems to be run from user space. From our point of view, FUSE has several significant benefits. First, with FUSE the bulk of the file system can be written in user space, speeding up development and reducing maintenance cost. Typically, in-kernel file systems take many years to mature, while FUSE-based file systems can do so in much shorter time. In fact, for researchers who are often not particularly kernel savvy, FUSE is the only choice to incorporate their research ideas into a working file system. Second, utilizing FUSE enables considerable code reuse since FUSE-based file systems can take advantage of existing libraries. The fact that FUSE-based file systems can be written in many languages (e.g., C, Java, and Python, to name a few) is an additional bonus.

A frequently voiced concern is that FUSE-based file systems suffer from lower performance compared to in-kernel file systems due to extra memory copies and context switching. However for our VVFS the relative ease of implementation provided by FUSE greatly outweighs any modest performance drawbacks. Likely, a similar cost-benefit analysis will hold true for other research driven file system development. We benchmarked FUSE on a small 8 core Linux system and found that FUSE overhead was not a performance limiting factor for our applications. In fact, our 
observed 4 gigabytes/second FUSE throughput was considerably faster than the 1.4 gigabytes/second of I/O 8-way parallel speed we obtained on our main Lustre based file system. In addition, FUSE-based file systems are often used in distributed environments in which any extra overhead is insignificant relative to overall system performance. However, if careful benchmarking revealed the existence of performance bottlenecks in the VVFS pipeline, the relevant code could be moved into kernel drivers. A case in point is the ZFS file system which was initially implemented on top of FUSE but later modified for in-kernel operation once the design was fully stable and its performance characteristics were known [8].

VVFS is currently under active development [9], and in the following case study we present our experience with an early development version applied to large-scale image data from electron microscopy.

\section{VVFS CASE STUDY: LARGE SCALE ELECTRON MICROSCOPY IMAGE STACKS}

An area of large-data science of particular interest to us and our collaborators is connectomics. Connectomics aims to determine the connectivity of neural tissue at levels ranging from functional circuits to whole brains. This requires full synaptic detail that can only be obtained with nanometer resolution electron microscopy $[10,11]$. The resulting datasets have data densities exceeding one petabyte per cubic millimeter of brain tissue and thus pose many computational and big data challenges. Our experience and struggles with processing a 32 terabyte electron microscopy dataset (Figure 3) of mouse visual cortex in collaboration with Davi Bock, Wei Chung Allen Lee and Clay Reid [12] motivated us to investigate more efficient and cost effective ways to process large multidimensional data volumes.

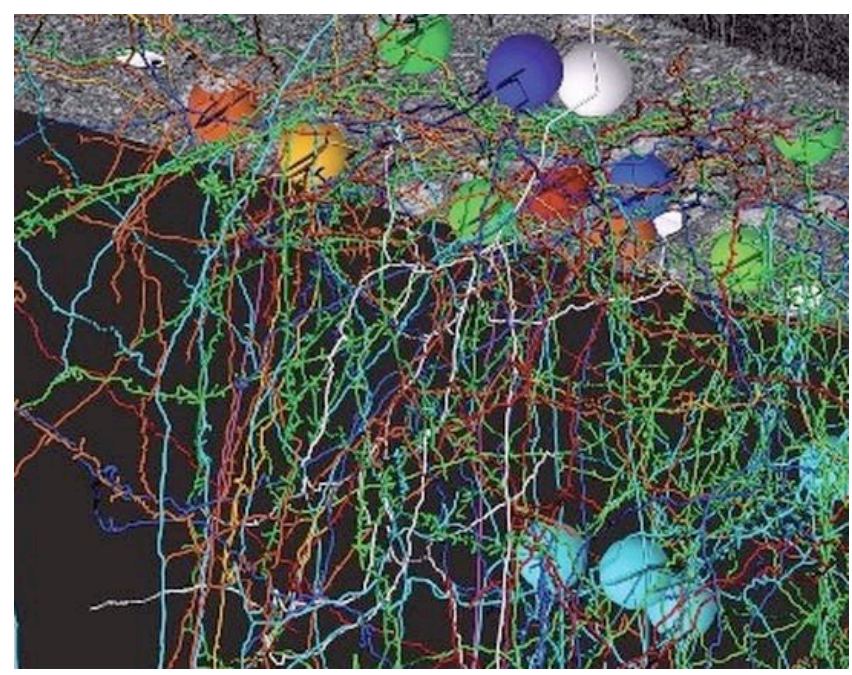

Figure 3. This colorized image shows local connections traced within a 10 TB mouse visual cortex electron microscopy dataset, aligned at the PSC and now being used as test data for VVFS development. Image courtesy of D. Bock and C. Reid.

The greatly reduced scale of the image shown in Figure 3 only hints at the size and complexity of this volume which was reconstructed from 3.2 million separate 10 megabyte images that had to be aligned both in 2D to form 100,000 by 80,000 pixel planes and in $3 \mathrm{D}$ to bring features through the third dimension into alignment. This process, carried out in 2009 by an early precursor to PSC's AlignTK software [13], had to correct for large compression and nonlinear distortions resulting from cutting ultrathin 40 nanometer sections (just a few hundred atoms thick) and also handled a range of defects including scratches, tears, debris, folds and occasional missing sections.

Neural pathways shown colorized in Figure 3 connect specific neurons of interest and are just a fraction of the structures in the data set. Tracing brain structures with long and complex branching connections requires both computational and interactive processing over many months and benefits from fast random access to arbitrary paths through a volume. Hence, random access retrieval and computational generation of oriented cuts through multi-terabyte virtual volumes has been our main initial focus and presents a simple but operationally significant benchmark of early VVFS development. We are currently working on providing efficient arbitrary cut-plane operation via VVFS and thus improve over and/or feed into tools like TrakEM2 [14] and CATMAID [15] that are commonly used for visual electron microscopy analysis but currently present views of pre-aligned images in the original planar orientation. The largest connectomics data sets, now in the 100-terabyte range, are expected to soon reach petabyte scales thanks to a new generation of parallel beam scanning electron microscopes [16]. Thus, near-data processing technologies like VVFS are desperately needed.

Within the VVFS pipeline we can leverage the performance characteristics of three different types of storage technologies: RAM, flash and disk, listed in order of decreasing performance. The rotational and seek delays of disk are particularly limiting, but its much lower cost and nonvolatility still make it the primary storage medium for multi-terabyte and petabyte applications. For VVFS cut-plane or slab generation it is critical to organize and selectively transfer data between storage systems in a way that maximizes delivery of virtual file content to end user applications; we are concerned with both bandwidth and latency. When the end use is a fully automated analysis procedure, we may not have firm limits on latency and may opt for maximum bandwidth. However, for interactive use it is important to provide fast and continuous response up to near video rate screen updates.

Conventionally, when flash storage is not available, arbitrary plane generation is optimized by careful layout of aligned data on disk using blocking schemes which improve storage locality over all three cardinal planes. This is usually done by using 3dimensional bricks containing small regions that are then linked into an octree, or by utilizing fractal-like spatial reorderings such as Morton or Hilbert curves [17]. Since flash memory is now a cost effective option to achieve truly random access at the block level, we can take advantage of it within a near-data server architecture.

To benchmark storage access vs latency and bandwidth for flash and disk drives, we measured generation of randomly oriented cut-planes through multiple 1 terabyte volumes organized in columnar fashion. Morton and Hilbert data orderings do not accelerate data access for flash storage, although they can still be useful for certain types of spatial search. As an indicator of performance, we plot in Figure 4 cut-plane pixels per second versus block size using single stream read and mmap access to both disk and flash storage.

The test configuration uses a 1.7 terabyte flash subsystem (Crucial_CT240M500SSD1) and a 260 terabyte Lustre file system running on Axon, a machine with four 3.3 GHZ Xeon E5-4627 processors with 8 cores each and 512GB shared memory. The peak rate of 5 Mpixels/second is for a single process generating independent random cut-planes from flash storage. Simultaneous 
access to simulate multi-user operation (not shown in the graph) scales well to an aggregate of $\sim 40$ Mpixels/second on our test

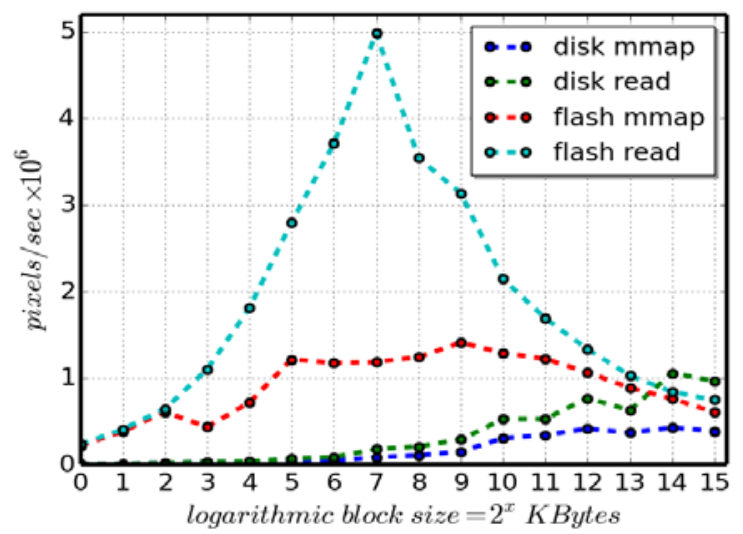

Figure 4. Benchmark of pixel rendering speed versus data record sizes for random polar cuts through 3D volumetric data. The curves represent read and mmap access to both flash and disk storage. For the best access mode, flash read, proper record size is critical to performance.

system until it saturates its raw data retrieval rate of $\sim 1.5$ GBytes/second. In real use scenarios arbitrary cut-plane accesses would be quickly followed by sequential steps to adjacent planes for video viewing, slab retrieval, or bulk computations such as maximum intensity projection. In those cases the majority of data will still be in memory, so the overhead of additional cuts will be low. Our measured rates show that an arbitrary view of a $1 \mathrm{Kx} 1 \mathrm{~K}$ cut appropriate for a visual display takes $\sim 200 \mathrm{~ms}$ with a single stream or $25 \mathrm{~ms}$ if 8 streams are merged using flash memory. The same operations from disk are an order of magnitude slower so there is clear benefit in mixing storage technologies for VVFS servers.

\section{FUTURE WORK AND CHALLENGES}

Several aspects of VVFS operation require further study. Using pathname encoding to describe virtual file content can result in a potentially unlimited number of virtual files (e.g. for continuous VVFS control parameters), and it is not immediately clear how to properly interface this with standard UNIX file system queries like ls. We expect that the VVFS control interface will handle many cases, but this will have to be tested in practice with a range of data types and applications. Another challenge is the ability to handle virtual files with lengths that are not known ahead of time, such as for compressed JPEG images. This may impact the operation of client programs that stat a virtual file before reading in order to allocate buffer space. Further, the length of virtual files as reported by ls can not be determined until the file contents are generated. In many cases, such as compressed images, client programs still operate correctly even if the size reported by stat is artificially inflated to some maximum size.

We have mainly focused on using the VVFS to provide read access for image processing applications since that has been our most urgent need. It is less clear what VVFS write operations should do. One useful case is to explicitly allow written content to override the usual virtual content when the file is next accessed, e.g. to allow for correction of damaged or missing blocks. We have used special control files to do on-the-fly manipulation of metadata by describing details of VVFS data manipulations including contrast and geometric transformations, but other methods and uses should be explored, as well as evaluating VVFS against other data access strategies.

\section{ACKNOWLEDGMENTS}

This work was supported by NIH award P41GM103712 and NSF award ACI1440756. We thank Greg Hood for technical discussions, R. Laghaei for help with preparing some of the figures, and our collaborators D. Bock, F. Engert, D. Hildebrand, and J. Lichtman for providing access to their large scale electron microscopy data.

\section{REFERENCES}

[1] Son, S.W., et al., 2010. Enabling Active Storage on Parallel I/O Software Stacks. in Proceedings IEEE Mass Storage Systems and Technologies, 2010.

[2] Piernas, J., et al., 2007. Evaluation of Active Storage Strategies for the Lustre Parallel File System. in Proc. ACM/IEEE SC'07

[3] Kurc, T. et al., Querying Very Large Multidimensional Datasets in ADR. in Proc ACM/IEEE SC'99, 1999.

[4] Beynon, M. et al., DataCutter: Middleware for Filtering Very Large Scientific Datasets on Archival Storage Systems. in IEEE Symposium on Mass Storage Systems, 2000

[5] Path and URL options: 2016. http://www.eww.com.hk/cfdocs/ Working_with_Verity_Tools/vspider7.html. Accessed: 2016- 06-15.

[6] ScriptFS: 2016. https://github.com/frodonh/scriptfs. Accessed: 2016- 06- 15.

[7] Libfuse: 2016. https://github.com/libfuse/libfuse. Accessed: 2016-06-15.

[8] ZFS: 2016. https://en.wikipedia.org/wiki/ZFS Accessed: 2016-06-15.

[9] AdaptFS: 2016. https://github.com/pscedu/adaptfs/ tree/master Accessed: 2016-06-15.

[10] Lichtman, J. et al. 2014. The big data challenges of connectomics. Nature Neuroscience. 17, 11 (2014), 1448-1454.

[11] Helmstaedter, M., Cellular-resolution Connectomics: Challenges of Dense Neural Circuit Reconstruction. Nature Methods 10, 501-507, 2013.

[12] Bock, D., et al., Network Anatomy and In Vivo Physiology of a Group of Visual Cortical Neurons. Nature, 471, 177182, 2011.

[13] AlignTK: 2016. http://mmbios.org/aligntk-home Accessed: 2016-06-15.

[14] TrakEM2: 2016. http://imagej.net/TrakEM2 Accessed: 2016-06-15.

[15] CATMAID: 2016. https://github.com/catmaid/CATMAID Accessed: 2016-06-15.

[16] Eberle, A. et al. 2015. Multiple-Beam Scanning Electron Microscopy. Microscopy Today. 23, 02 (2015), 12-19.

[17] Bohm, C., et al., XZ-Ordering: A Space-Filling Curve for Objects with Spatial Extension. Lecture Notes in Computer Science, 1651, 75-90, 1999. 